\title{
Present and Active: Transglutaminases in the Virgin Rat Uterus and Cervix
}

\author{
Lindsey W. Young ${ }^{1, *}$, Janice M. Thompson $^{1}$, Kiyotaka Hitomi $^{2}$ and Stephanie W. Watts ${ }^{1}$ \\ ${ }^{\text {I}}$ Pharmacology and Toxicology, Michigan State University, East Lansing, MI, USA \\ ${ }^{2}$ Graduate School of Pharmaceutical Sciences, Nagoya University, Japan, Research Conducted at Michigan State \\ University, Funded in part by NIH R25 HL103156 and R01 HL107495
}

\begin{abstract}
Transglutaminases are a family of enzymes that are known for their protein cross-linking abilities. Transglutaminases have been investigated very little in female reproductive tissues. We hypothesized that transglutaminase proteins were present and active in the virgin rat uterus and cervix. In both tissues, real time RT-PCR identified transglutaminase 1,2,3, and 4 mRNA, and immunohistochemistry determined the presence of transglutaminase 1, 2, 3, and 4 proteins. Transglutaminase 1,2 and 3 were active in the virgin rat uterus, and transglutaminase 1 and 3 were active in the virgin rat cervix, identified in situ by isoform-specific FITC-labeled substrates. Transglutaminase 2 was active in the virgin rat uterus but absent in the cervix. The transglutaminase inhibitor cystamine ( $1 \mathrm{mM})$ reduced maximal agonist-induced isometric contraction in the uterus, but not the cervix. This study provides new knowledge of active transglutaminase isozymes in female reproductive tissues.
\end{abstract}

Keywords: Cervix, contractility, cystamine, reproduction, transglutaminase, uterus.

\section{INTRODUCTION}

Transglutaminases (TGs) are a family of enzymes identified by their ability to modify proteins by cross-linking [creating epsilon-(gamma-glutamyl) lysine isopeptide bonds [1]], often in the presence of elevated $\mathrm{Ca}^{2+}$ [1-3]. Protein cross-linking is the major function of TG investigated, but the post-translational modification of proteins by TG (such as deamidation and amine incorporation [4]) has also been studied, as well as kinase [1] and GTPase activity [2]. While TGs are implicated in neurodegenerative diseases [4, 5], minimal information is known about TG in reproductive tissue.

Eight members of the TG family are catalytically active in mammals: TG1 through TG7, and blood coagulation factor XIII [2]. TGs have been identified in the skin epidermis [6], brain [5], prostate [7], and saliva [8], but not in female reproductive tissue (which throughout this study will refer exclusively to the uterus and cervix). TGs have been implicated in contraction of the vasculature [9]. Uterine smooth muscle contractility is important for implantation, parturition, embryo transport, and many other functions in the estrous cycle [10], but very little is known about the function of cervical smooth muscle. We hypothesized that TGs would be present and active in the virgin rat uterus and cervix, and would play a role in uterine and cervical contractility.

*Address correspondence to this author at the Dept. of Pharmacology and Toxicology, Michigan State University, B445 Life Sciences, 1355 Bogue Street, East Lansing, Michigan, USA 48824; Fax: 517 353-8915;

E-mail: youngli9@msu.edu
Our study addressed the question of the presence and activity of TGs in female reproductive tissues in several ways. First, the presence of TG mRNA in the virgin rat uterus and cervix was explored. Second, the sites of expression and activity for TG protein were determined using immunohistochemistry and newly developed isoformspecific TG substrates. Lastly, the ability of observed TGs to serve the process of smooth muscle contraction in virgin reproductive tissues was determined by using isolated tissue baths to measure isometric contraction.

\section{MATERIALS AND METHODOLOGY}

\section{Animal Use}

The Michigan State University Institutional Animal Care and Use Committee approved each of the following protocols. Virgin female Sprague-Dawley rats between 1012 weeks of age (Charles River Laboratories, Portage, MI) were used for all experiments, and they were freely cycling. Rats were euthanized by $60 \mathrm{mg} / \mathrm{kg}$ pentobarbital (i.p.). In all rats, the ovaries, uterine horns, cervix, and vagina were removed as a whole and placed in a beaker containing physiological salt solution [PSS, $\mathrm{mM}$ : $\mathrm{NaCl}, 130 ; \mathrm{KCl}, 4.7$; $\mathrm{KH}_{2} \mathrm{PO}_{4}, 1.18 ; \mathrm{MgSO}_{4} .7 \mathrm{H}_{2} \mathrm{O}, 1.17 ; \mathrm{NaHCO}_{3}, 14.8$; dextrose, 5.5; $\mathrm{Na}_{2}$ EDTA. $\left.2 \mathrm{H}_{2} \mathrm{O}, 0.03 ; \mathrm{CaCl}_{2}, 1.6 ;(\mathrm{pH}=7.2)\right]$. Tissues were then used in one of the protocols described below.

\section{Real-Time RT-PCR}

The cervix and right uterine horn were removed from anesthetized rats. The MELT Total Nuclei Acid Isolation System (cat. no. AM1983,Ambion, Austin, TX) was used to 
isolate RNA from each sample and 0.8-1 $\mu \mathrm{g}$ RNA from each sample was reverse transcribed with Superscript II reverse transcriptase (cat. no. 18064-014, Invitrogen, Carlsbad, CA). A GeneAMP PCR System 7500 Fast (Applied Biosystems, Carlsbad, CA) and SYBR Green PCR Master Mix (Applied Biosystems) were used to complete standard real-time RTPCR. B-2 microglobulin (B2M) was used as the calibrator gene. Rat primers were purchased from RealTimePrimers.com (B2M, accession no. NM_012512; Tgm1, accession no. NM_031659; Tgm2, accession no. NM_019386; Tgm3, NM_001108959; Tgm4, NM_022713; Tgm5, NM_201631; Tgm7, XM_002726167). The following PCR conditions were used: $95^{\circ} \mathrm{C}$ for $10 \mathrm{~min}$ followed by 50 cycles $\left(95^{\circ} \mathrm{C}, 10 \mathrm{sec} ; 58^{\circ} \mathrm{C}, 45 \mathrm{sec}\right)$. A standard dissociation curve was run upon completion of the above cycle conditions as per the SA Biosciences SYBR Green protocol.

\section{Histology}

The uterus and cervix were cleaned of fat, and sectioned to a $5-10 \mu \mathrm{m}$ thickness by the Investigative Histopathology lab at Michigan State University. For immunohistochemistry, the uterus was cut open longitudinally and cross-sections of that strip were mounted on slides. For the TG activity assay, closed cross-sections of the uterine horn were mounted. Closed cross-sections of the cervix were mounted on slides for both immuno-histochemistry and the TG activity assay. Tissues for immuno-histochemistry were formalin-fixed and paraffin-embedded, while tissues for the activity localization assay were fresh-frozen and saved in a $80^{\circ} \mathrm{C}$ freezer until needed.

\section{Immunohistochemistry}

Paraffin-embedded tissue sections were dewaxed (Histochoice Clearing Agent, H-2779, Vector Laboratories, Burlingame, CA, USA; $100 \%$ isopropanol, $\mathrm{dH}_{2} \mathrm{O}$ ) and unmasked (Antigen Unmasking Solution, H-3300, Vector). Slides were rinsed with $\mathrm{dH}_{2} \mathrm{O}$, blocked with $0.3 \% \mathrm{H}_{2} \mathrm{O}_{2} / \mathrm{PBS}$, rinsed twice with Dulbecco's Phosphate Buffered Saline (PBS, Product no. D-8537, Sigma-Aldrich, St. Louis, MO, USA) for $5 \mathrm{~min}$ per wash, and blocked with species-specific blocking serum $(1.5 \%$, made in PBS) for $30 \mathrm{~min}$ at room temperature. Blocking serum was removed from one half of the sections (sections were present in duplicate), and primary antibody (mouse monoclonal anti-transglutaminase 1, cat. no. sc-166467, Santa Cruz Biotechnology, Dallas, TX, USA; mouse monoclonal anti-transglutaminase 2, cat. no. MS-279R7, Neomarkers Inc., Fremont, CA, USA; goat polyclonalanti-transglutaminase 3, cat. no. sc-23365, Santa Cruz Biotechnology, Dallas, TX, USA; mouse monoclonal, anti-transglutaminase 4, ATGen, cat. no. ATGA0140, made up in $1.5 \%$ blocking serum) was applied at a $5 \mu \mathrm{g} / \mathrm{mL}$ concentration to the other half, such that there was an identical section present without the primary antibody. As positive controls, human skin (cat. no. 12-701-XA1; ProSci, Loveland, CO, USA) was used for TG1 and TG3, rat vena cava (paraffin-embedded and sectioned by Investigative Histopathology of Michigan State University) was used for TG2, and human prostate was used for TG4 (cat. no. 10-635YA1, ProSci, Loveland, CO, USA). Slides were incubated at $4^{\circ} \mathrm{C}$ overnight. The kits and reagents used in the remainder of this protocol were from Vector Laboratories (Burlingame,
CA, USA). Primary antibody or blocking serum was removed from the sections, and secondary antibody $(10 \mathrm{~mL}$ PBS, $1.5 \%$ blocking serum, TG1, TG2, and TG4, PK-6102; TG3, PK-6105) was applied to all sections for $30 \mathrm{~min}$ at room temperature in a humidified chamber. Sections were incubated in ABC Elite Reagent (cat. no. PK-6100) for 30 $\mathrm{min}$ at room temperature in a humidified chamber. Sections were then washed with PBS (3 times, 5 min each) and incubated with ImmPAC TDAB substrate (cat. no. SK-4105) for $5 \mathrm{~min}$. Sections were washed again with PBS (3 times, 5 min each), counterstained with Nuclear Fast Red (cat. no. H3403), mounted with Vectamount (permanent H-5000 or aqueous H-5501), and covered with a glass coverslip. Slides were photographed on a Nikon Eclipse inverted microscope with a Nikon Digital Sight DS-Qil camera, and images were captured within MMI Cell tools (MMI, Eching, Germany, version 3.47). Images were not altered except to universally adjust brightness and contrast.

\section{In Situ Activity Localization Assay}

Fresh-frozen tissue sections (5-10 $\mu \mathrm{m}$ thickness) were used, and exposed to TG1-specific (K5), TG2-specific (T26), or TG3-specific (E51) FITC-labeled peptides, or their respective inactive QN controls. The K5QN, T26QN, and E51QN control peptides (containing an asparagine $(\mathrm{N})$ residue instead of the glutamine (Q) present in the experimental K5, T26, and E51 peptides) cannot act as substrates for the TG family, which cross-link glutamine and lysine residues. Rat and mouse aorta were used as controls in our lab to determine viability for K5 (TG1) and T26 (TG2), respectively. Rat skin was used as a positive control for E5 1 (TG3) experiments. Tissues were stored at $-80^{\circ} \mathrm{C}$ until they were ready for use. The sections were removed from the freezer and warmed to room temperature for $10 \mathrm{~min}$. Sections were incubated in blocking solution [1\% albumin from bovine serum (BSA), cat. no. A2153-50G, SigmaAldrich, St. Louis, MO, USA; $150 \mathrm{mM} \mathrm{NaCl}, \mathrm{PBS}]$ at room temperature for $30 \mathrm{~min}$, and removed using a blotting technique. Substrate reaction solution $\left(\mathrm{dH}_{2} \mathrm{O}, 5 \mathrm{mM} \mathrm{CaCl}\right.$, $100 \mathrm{mM}$ Tris-Cl, $1 \mathrm{mM}$ DTT, and $0.001 \mathrm{mM}$ FITC peptide (K5 [YEQHKLPSSWPF] or K5QN for TG1 slides [11], T26 [HQSYVDPWMLDH] or T26QN for TG2 slides [12], E51 [PPPYSFYQSRWV] or E51QN for TG3 slides [13], developed and supplied by Kiyotaka Hitomi) was applied for 90 min at $37^{\circ} \mathrm{C}$, covered in a light-tight box, and humidified by a wet sponge.

Substrate solutions were removed and stop solution (PBS, $25 \mathrm{mM}$ EDTA) was added to the sections for $5 \mathrm{~min}$. Stop solution was then removed and sections were washed with PBS. Prolong Gold with DAPI (Invitrogen, Carlsbad, CA, U.S.A.) was applied. Images were visualized on a Nikon Eclipse inverted fluorescence microscope and were captured within Nikon NIS elements BR 3.00 software. Images were not altered when overlays were created, and treatment and control slides were imaged using the same DAPI and FITC exposure time $(2.5 \mathrm{sec}$ to $4 \mathrm{sec}$ for $4 \mathrm{x}$ images, $150 \mathrm{~ms}$ to $2 \mathrm{sec}$ for $2 \mathrm{x}$ images) and LUT (look up table) settings.

\section{Isometric Contractility}

Rat uterine horns and cervices were dissected and cleaned of fat in PSS. The horns were then cut into 
TG in Virgin Rat Uterus and Cervix

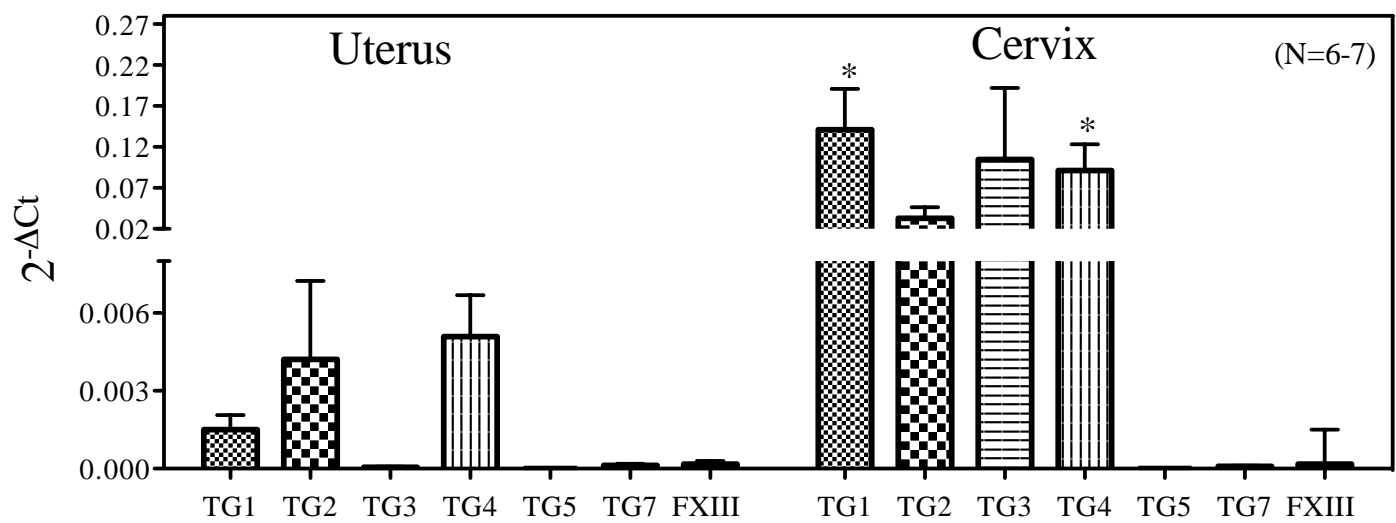

Fig. (1). Real Time RT-PCR. Standard real-time RT-PCR analysis of transglutaminase mRNA expression in the virgin rat uterus and cervix $(n=6-7)$. Bars represent means \pm SEM for the number of animals in parentheses. $*$ represents $p$-value $<0.05$, comparing the amount of mRNA for each isozyme in the cervix to the uterus, relative to B2M.

longitudinal strips ( $2 \mathrm{~cm}$ long, $0.5 \mathrm{~cm}$ wide), and mounted using silk suture. The cervix (notably white) was cleaned of fat, separated from the uterine body and the vagina, and Lshaped hooks were placed within the lumens. Uterine strips (longitudinal preparation) and cervical rings (circular preparation) were then mounted in warmed, aerated PSS $\left(37^{\circ} \mathrm{C} ; 95 / 5 \% \mathrm{O}_{2} / \mathrm{CO}_{2}\right)$ in isolated tissue baths $(30 \mathrm{~mL})$ for measurement of isometric contractile force (Grass Instruments displacement transducer, Grass Instruments, Quincy, MA), using Power Lab(model 4, AD Instruments inc., CO, USA) and a Quad bridge connected to a Mac (AD Instruments). The tissues were placed under optimum resting tension (1 g, previously determined [14]) and initially challenged with $100 \mathrm{mM}$ potassium chloride $(\mathrm{KCl})$ to test for tissue viability. Tissues were washed every $15 \mathrm{~min}$ until they returned to resting tension. Cystamine (at a concentration known to inhibit transglutaminase activity [9], $1 \mathrm{mM}$, cat. no. C8707-25G, Sigma-Aldrich, St. Louis, MO, USA), or its vehicle $\left(\mathrm{H}_{2} \mathrm{O}\right)$ was incubated with the tissues for 1 hour. This incubation was followed by a cumulative concentration response curve to carbamylcholine, during which tissues were incubated in each concentration for $5 \mathrm{~min}\left(10^{-9} \mathrm{M}\right.$ to $3 \mathrm{x}$ $10^{-4} \mathrm{M}$, cat. no. C-4382, Sigma-Aldrich, St. Louis, MO, USA). Cystamine $\left(\mathrm{NH}_{2}-\left(\mathrm{CH}_{2}\right)_{2}-\mathrm{S}-\mathrm{S}-\left(\mathrm{CH}_{2}\right)_{2}-\mathrm{NH}_{2}\right)$ is reduced to cysteamine intracellularly; cysteamine competitively inhibits $\left(\mathrm{K}_{\mathrm{i}} \sim 10^{-4} \mathrm{M}\right)$ transglutaminase in a general manner by acting reversibly as an alternate amine substrate $[15,16]$. Tissues were again exposed to $\mathrm{KCl}$ to determine that their viability was equivalent to the beginning of the experiment.

\section{Data Analyses}

All data are presented as means \pm SEM (standard error of the mean). Data for real time RT-PCR were calculated as the mean of the $2^{-\Delta \mathrm{CT}}$ values \pm the SEM, and statistical significance ( $\mathrm{p}$-value $<0.05$ ) was determined by an unpaired t-test. Activity localization assay data was quantified by densitometric analysis as follows (Image J, version 1.46e). Three images were captured of each area listed here (uterus: epithelial lining of the endometrium (EL), endometrium (E), inner circular myometrium (C), outer longitudinal myometrium (LM), perimetrium (P); cervix: epithelium (EP), subepithelial stroma (S), inner muscular stroma (I), outer muscular stroma (O)) and the area of the section $\left(\mu \mathrm{m}^{2}\right)$ was measured by the polygon tool of Image J. Different magnifications were normalized by determining the number of pixels $/ \mu \mathrm{m}$. The mean signal intensity (represented in arbitrary densitometry units $\mathrm{px} / \mu \mathrm{m}$ ) was divided by the average area of the signal $\left(\mu \mathrm{m}^{2}\right)$ to determine the signal density $\left(\mathrm{px} / \mu \mathrm{m}^{3}\right)$. The signal density of the peptide and peptide control (QN, containing asparagine [N] instead of the necessary glutamine [Q]) were then compared via a Mann-Whitney U nonparametric test, and statistical . Due to the oscillatory nature of uterine and cervical contraction, isometric data were reported as the integration (LabChart 7.0, ADInstruments Ltd.) of the activity in 5-min intervals as previously described [14]. Integrated units were then normalized to the initial $\mathrm{KCl}$ contraction. A two-way ANOVA and Bonferroni's post-hoc multiple-comparison test (GraphPad Prism 6.0, GraphPad Software, La Jolla, CA) were used to compare individual data points in the concentration response curve to their corresponding control, and statistical significance was stated if $\mathrm{p}$-value $<0.05$.

\section{RESULTS}

\section{Transglutaminase mRNAs are Present in the Virgin Rat Uterus and Cervix}

Real-time RT-PCR was used to investigate the presence of mRNA for TG1 through TG5, TG7, and Factor XIII. mRNA for TG1, TG2, TG3, and TG4 were present in both the uterus and cervix (Fig. 1). The cervix had significantly more TG1 and TG4 mRNA expression relative to B2M than the uterus $(\mathrm{p}<0.05$, unpaired $\mathrm{t}$-test $)$. TG3 expression was minimal in the uterus, but was expressed in the cervix. Differences in TG2, TG7, and Factor XIII mRNA expression between the uterus and cervix (relative to B2M) were statistically insignificant. mRNA expression of TG5 was not observed in either the rat uterus or cervix. 

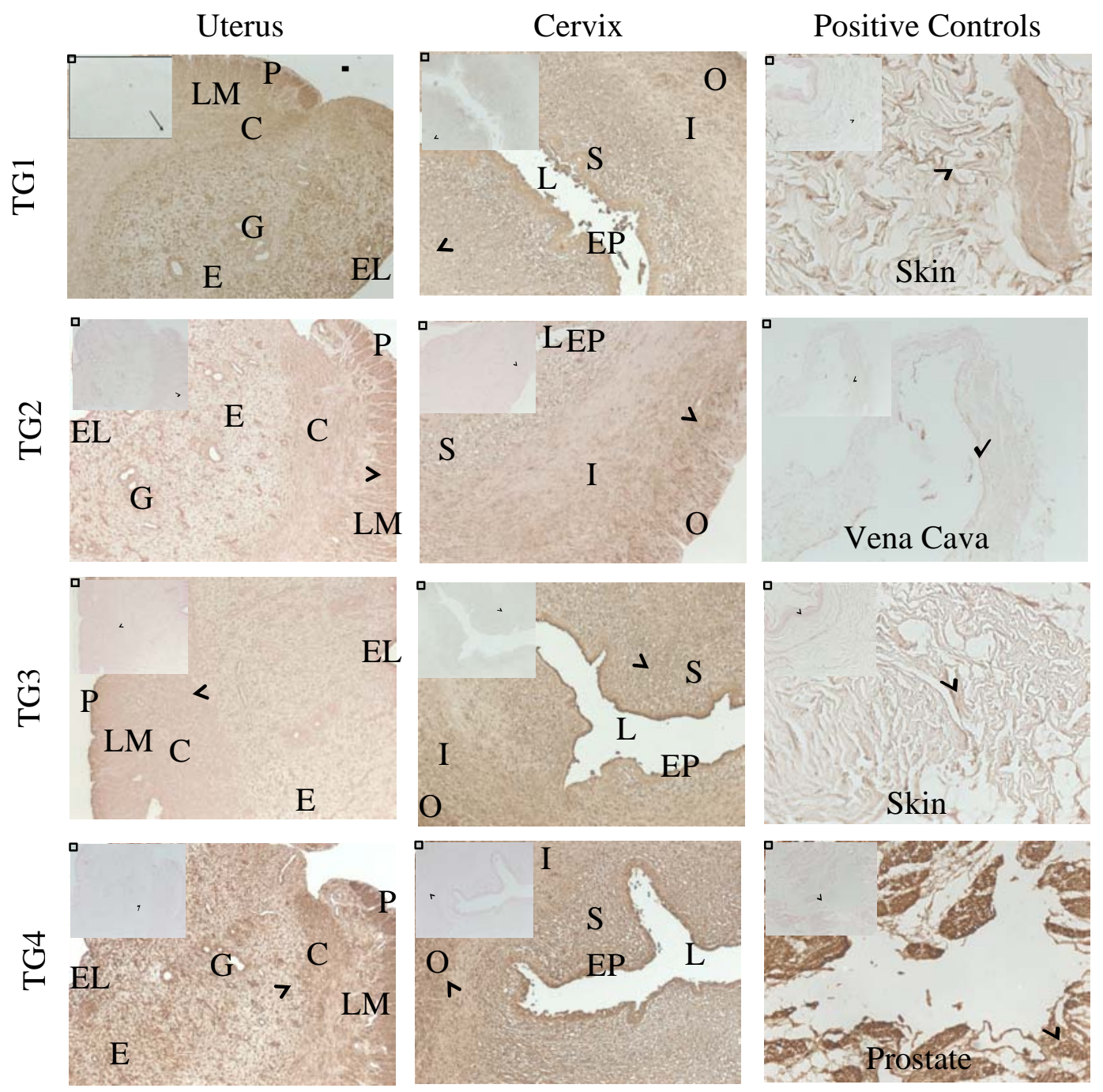

Fig. (2). Brightfield immunohistochemical staining of the virgin rat uterus (left panel) and virgin rat cervix (middle panel) with respective controls (right panel). Experimental images are paired with their control [no primary antibody added (most upper left)]. Red indicates the nuclear marker, while brown indicates the presence of the protein via the combination of the primary and secondary antibodies. Images were captured at a 10x magnification. $\mathrm{n}=7$ for all tissues. In the uterus, EL=epithelial lining of the endometrium, $\mathrm{E}=\mathrm{endometrium}, \mathrm{C}=\mathrm{inner}$ circular layer of the myometrium, LM=outer longitudinal layer of the myometrium, $\mathrm{P}=$ perimetrium. In the cervix, $\mathrm{L}=\mathrm{lumen}, \mathrm{EP}=\mathrm{epithelium}$, $\mathrm{S}=$ subepithelial stroma, $\mathrm{I}=$ inner muscular stroma, $\mathrm{O}=$ outer muscular stroma. The horizontal bar indicates a length of approximately 100 microns for all images. Arrows point to areas of interest. Positive controls for both the uterus and cervix (rat skin for TG1 and TG3, vena cava for TG2, and human prostate for TG4) are included in the right-most panel.

\section{Transglutaminase 1, 2, 3, and 4 Proteins are Present in the Virgin Rat Uterus and Cervix}

We next investigated the presence of the TG isoforms detected by RT-PCR via immunohistochemical analysis. TGs 1, 2, 3, and 4 were present in both the uterus (Fig. 2, left-most panel) and cervix (Fig. 2, middle panel). Staining was absent on slides lacking the primary antibody (insert in upper left hand corner). All tissues were counterstained with Nuclear Fast Red. Skin was used as a positive control for TG1 and TG3, vena cava was the positive control for TG2, and human prostate was used as a control for TG4 (all Fig. 2, right-most panel). Arrows indicate areas of interest.

In the uterus (Fig. 2, left-most panel), TG1 was present throughout all of the layers [the endometrium (E), inner circular myometrium (C), outer longitudinal myometrium $(\mathrm{LM})$, and perimetrium $(\mathrm{P})]$, in the epithelial lining of the endometrium (EL), and around the endometrial glands (G). Arrows indicate areas of interest. TG2 was consistently present throughout the endometrium (E) and longitudinal myometrium (LM), but was variably expressed in the 
Uterus
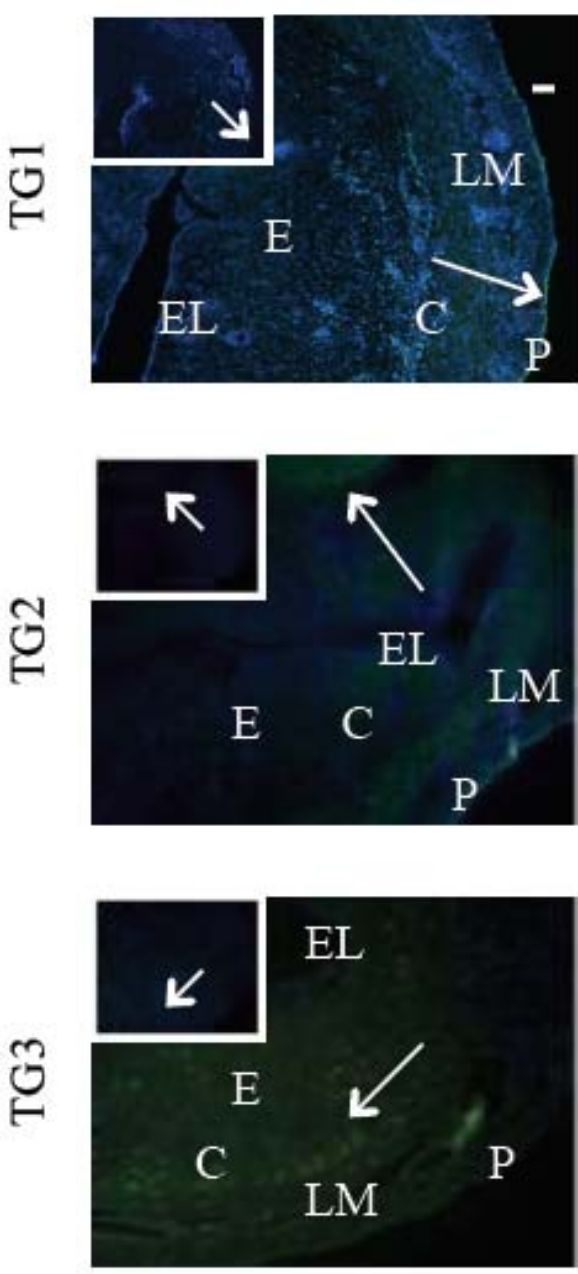

Cervix
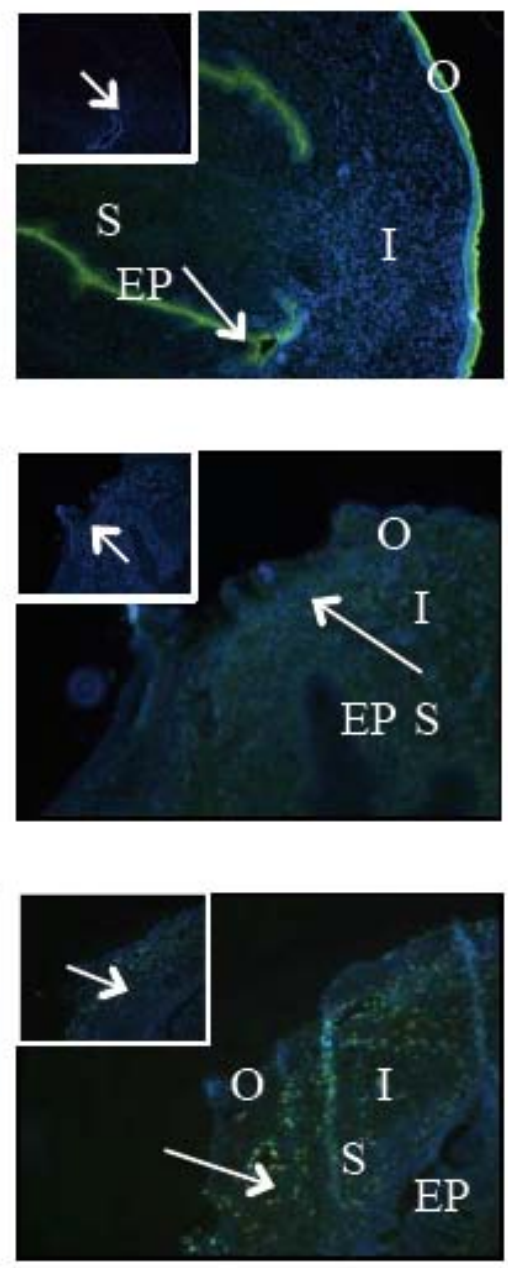

Positive Controls
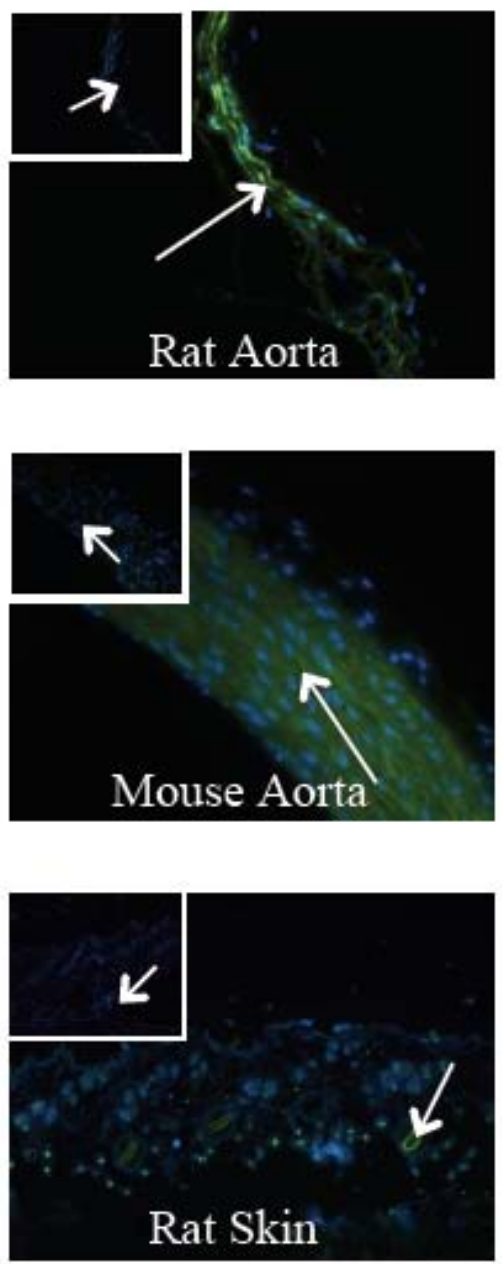

Fig. (3). In situ TG1, TG2, and TG3 activity localization assay of the virgin rat uterus (left panel) and virgin rat cervix (middle panel) with respective controls (right panel). Experimental images of the rat uterus (K5 for TG1, T26 for TG2, or E51 for TG3) are paired with the control inactive peptide (K5QN for the TG1 control, T26QN for the TG2 control, or E51QN for the TG3 control, most upper left) images, at a 4x magnification. These images are representative of $n=5-7$ for the uterus, $n=7$ for the K5 and T26 cervix, and $n=6$ for the E51 cervix In the uterus, $\mathrm{EL}=$ epithelial lining of the endometrium, $\mathrm{E}=$ endometrium, $\mathrm{C}=$ inner circular layer of the myometrium, $\mathrm{LM}=$ outer longitudinal layer of the myometrium, $\mathrm{P}=$ perimetrium. In the cervix, $\mathrm{L}=$ lumen, $\mathrm{EP}=$ epithelium, $\mathrm{S}=$ subepithelial stroma, $\mathrm{I}=$ inner muscular stroma, $\mathrm{O}=$ outer muscular stroma. Nuclei (blue) were identified using DAPI. The horizontal white bar indicates a length of approximately 100 microns in all images. Positive controls for both the uterus and cervix (rat aorta for TG1, mouse aorta for TG2, and rat skin for TG3) are included in the rightmost panel.

epithelial lining of the endometrium (EL), endometrial glands $(\mathrm{G})$, circular myometrium $(\mathrm{C})$, and perimetrium $(\mathrm{P})$. TG3 was consistently located throughout the longitudinal myometrium (LM) and the perimetrium (P), but was variably expressed in the epithelial lining of the endometrium (EL), the endometrium $(\mathrm{E})$, the endometrial glands $(\mathrm{G})$, and the circular myometrium (C). TG4 was consistently present throughout all regions that we have identified in this study.

In the cervix (Fig. 2, middle panel), TG1 was present throughout the epithelium (EP), subepithelial stroma (S), inner muscular stroma (I) and outer muscular stroma (O). TG2 was consistently present in the outer muscular stroma (O), and the epithelium (EP), and was variably expressed in the inner muscular (I) and subepithelial stroma (S). TG3 proteins were variably expressed in all regions. TG4 protein was present throughout the epithelium (EP), subepithelial stroma (S), inner muscular stroma (I) and outer muscular stroma $(\mathrm{O})$.

\section{Transglutaminases are Active in the Virgin Rat Uterus and Cervix}

To determine if TG1, TG2, and TG3 were functionally active in the rat uterus and cervix, we took advantage of isoform-specific peptides recognized by reactive $\mathrm{TG}$ enzymes. Fresh frozen uterine and cervical sections were exposed to TG1-specific (K5), TG2-specific (T26), and TG3-specific (E51) FITC-labeled peptides. TG4 was not investigated because a specific peptide for this isozyme was not available at the time of experimentation. Images (Fig. 3) show tissues that were incubated with the experimental FITC-peptides, while images in the upper left hand corner show tissues that had control QN-peptides applied at an 
Table 1. Difference in average transglutaminase signal densities in the uterus (active peptide signal density minus inactive peptide signal density, \pm SEM). Active peptides $(\mathrm{K5}, \mathrm{T26}$, E51) contain the glutamine residue required for transglutaminase activity, while inactive peptides contain an asparagine, rendering transglutaminase inactive. P-values (beneath the dotted line) were calculated by a Mann-Whitney $U$ nonparametric test. P-values compare transglutaminase activity signal in the tissues exposed to the active peptide vs. the inactive peptide. P-values $<0.05$ were considered significant. Significance is emphasized with italics and bold. $\mathrm{N}=5-7$.

\begin{tabular}{|c|c|c|c|}
\hline & & & \\
\hline
\end{tabular}

equivalent concentration. The K5QN, T26QN, and E51QN control peptides (containing an asparagine residue $(\mathrm{N})$ instead of the glutamine (Q) present in the experimental K5, T26, and E51 peptides) cannot act as substrates for the TG family, which cross-link glutamine and lysine residues. Positive controls for the TG activity localization were the rat aorta, mouse aorta, and rat skin for TG1, TG2, and TG3 respectively (Fig. 3, right-most panel). Arrows point to areas of interest. There was modest punctate fluorescent staining in some images from the control; therefore enzymatic activity was reported only if the averaged quantity of fluorescence in the experimental slides (containing the glutamine required for TG to function) was significantly different than that of the asparagine controls in corresponding areas. Table $\mathbf{1}$ shows the Mann-Whitney U nonparametric test results in the uterus, comparing TG signal density in the active peptide to that of the inactive peptide. Table 2 shows the Mann-Whitney U nonparametric test results in the cervix.

In the rat uterus (Fig. 3, left-most panel), TG1 activity (pvalue $<0.05$, Mann-Whitney $U$, Table 1) was observed throughout the epithelial lining of the endometrium (EL), inner circular myometrium (C), and perimetrium (P). TG2 activity (p-value $<0.05$, Mann-Whitney $U$, Table 1) was significant in the endometrium (E), inner circular myometrium (C), and outer longitudinal myometrium (LM) of the uterus. TG3 was active (p-value $<0.05$, Mann-Whitney $\mathrm{U}$, Table 1) in the endometrium (E), inner circular myometrium (C), outer longitudinal myometrium (LM), and perimetrium $(\mathrm{P})$.
In the rat cervix (Fig. 3, middle panel), TG1 was active (p-value $<0.05$, Mann-Whitney U, Table 2) in the epithelium (EP) and the outer muscular stroma (O). When fluorescence between images was averaged, TG2 was not significantly active (p-value $>0.05$, Mann-Whitney $U$, Table 2) in any areas when compared to the control QN slide. TG3 was active (p-value $<0.05$, Mann-Whitney U, Table 2) in the subepithelial stroma (S) and outer muscular stroma (O).

\section{Transglutaminase Inhibition Reduces Uterine Contrac- tion}

An isolated tissue bath system was used to measure the isometric contraction of rat uteri and cervices when exposed to a general TG inhibitor, cystamine (Fig. 4). Carbamylcholine (carbachol) was selected as a cholinergic muscarinic agonist [17] to contract the uterus and cervix, which are innervated by the parasympathetic nervous system in which acetylcholine acts as a neurotransmitter [18, 19]. Carbachol induced uterine and cervical contraction when added in a cumulative fashion (ranging from $10^{-9} \mathrm{M}$ to $3 \times 10^{-4} \mathrm{M}$ ). Compared to tissues incubated with vehicle, cystamine inhibited uterine and cervical contraction to carbachol. However, this inhibition was quantitatively different between tissues. Maximum contraction was reduced by cystamine in the uterus but not the cervix (uterus $=43.70 \% \pm$ $7.97 \%$ vehicle contraction, $\mathrm{p}<0.05$ for concentrations $3 \times 10^{-5}$ to $3 \times 10^{-3} \mathrm{M}$, cervix $=89.00 \% \pm 9.76 \%$ vehicle contraction, p>0.05 for all concentrations except $10^{-6} \mathrm{M}$, two-way ANOVA). 
Table 2. Difference in average transglutaminase signal densities in the cervix (active peptide signal density minus inactive peptide signal density, \pm SEM). Active peptides $(\mathrm{K5}, \mathrm{T26}$, E51) contain the glutamine residue required for transglutaminase activity, while inactive peptides contain an asparagine, rendering transglutaminase inactive. P-values (beneath the dotted line) were calculated by a Mann-Whitney $U$ nonparametric test. P-values compare transglutaminase activity signal in the tissues exposed to the active peptide vs. the inactive peptide. $P$-values $<0.05$ were considered significant. Significance is emphasized with italics and bold. $\mathrm{N}=5-7$.

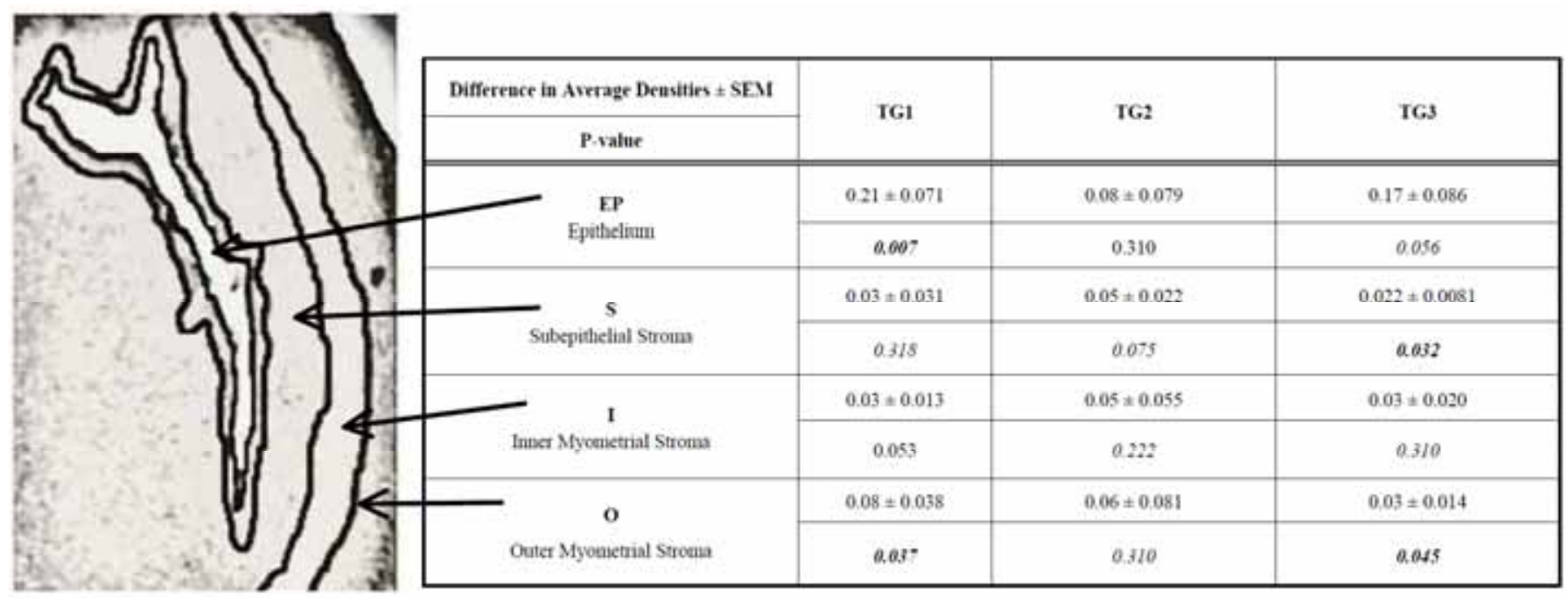
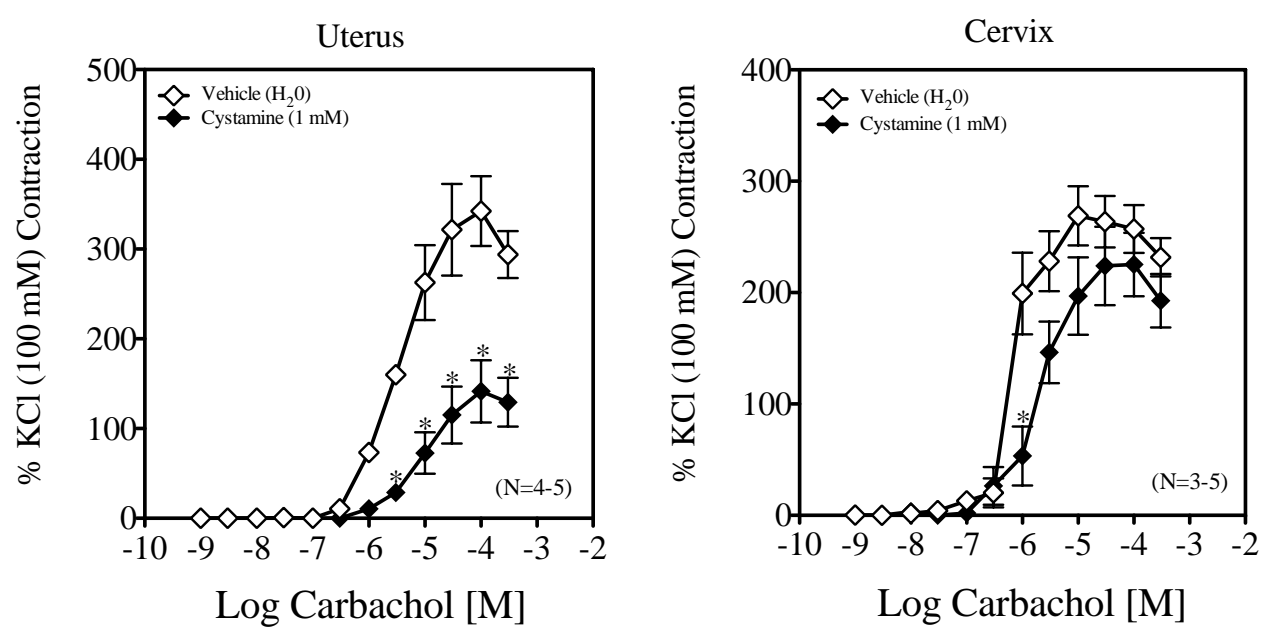

Fig. (4). Measurement of carbachol-induced contraction in virgin rat uterus and cervix exposed to vehicle (H2O) or cystamine (1 mM). Vehicle or cystamine were incubated with tissue for $1 \mathrm{~h}$ prior to agonist exposure. This incubation was followed by a cumulative concentration response curve during which tissues were incubated in each concentration for $5 \mathrm{~min}$. Points represent mean $\pm \mathrm{SEM}$ for the $N$ indicated. $\mathrm{KCl}=$ potassium chloride. * represents $\mathrm{p}<0.05$ versus the vehicle for each concentration in the curve (two-way ANOVA). N=4-5 animals for the uterus, $n=3-5$ animals for the cervix.

\section{DISCUSSION}

Though TGs have been studied in neurodegenerative [5] and skin diseases [20], TG expression and activity in reproductive tissues such as the uterus and cervix is comparatively understudied. The main goal of our research was to determine if TGs were present, active, and functional in the virgin rat uterus and cervix. Immunohistochemistry and a TG activity assay allowed us to identify the distinct expression and activity patterns of the different isozymes in these reproductive tissues, information that Western Blot analysis would not provide.

\section{Transglutaminases were Present and Active in the Virgin Rat Uterus and Cervix}

In this study, TG1, TG2, TG3, and TG4 were expressed in the rat uterus and cervix. These findings complement those made by others, namely the presence of TG1 in human cervical epithelial cells [21], the upper layers of the normal human cervix [22], and in human cervix carcinomas [22]. The TG2 expression that we observedin the rat cervix contrasts with a study conducted by Del Nonno et al. in which TG2 was not expressed in the human cervix, except in premalignant lesions in the epithelium caused by human 
papillomavirus infection [23]. These experiments only identified the presence of a protein recognized by antibodies, and thus we took a novel step in determining whether the identified TGs were active.

\section{Transglutaminase 2 is Active in the Virgin Rat Uterus, not the Cervix}

This study identified TG2 activity as a major difference between the rat uterus and cervix; several lines of evidence point to this conclusion. TG2 was active in the uterus [endometrium (E), circular myometrium (C), and longitudinal myometrium (LM)] but was not active in the cervix (as identified by lack of incorporation of the TG2specific peptide substrate T26 at a level significantly greater than the control peptide). Cystamine, a general TG inhibitor, inhibited maximum isometric contraction of the uterus to carbamylcholine $(\mathrm{p}<0.05$, Fig. 4), but did not reduce maximal contraction of the cervix. We speculate, then, that TG2 may mediate uterine contraction, at least to carbamylcholine. This idea is supported by a study by Alcock et al. in which TG2 inhibition by cystamine successfully inhibited carbamylcholine-induced contractility in the human myometrium [24]. Expanding upon these results may open new doors for understanding how the uterus and cervix work physiologically, and how to discriminate between the two tissues pharmacologically. The question remains as to why TG2, while identified by immunohistochemistry, was not active in the cervix. Endogenous inhibitors of TG2 (such as nitric oxide [25]) could be present in a greater magnitude in the cervix than the uterus, and this could be a reason behind the lack of activity of TG2 in the cervix.

While TG2 plays a role in uterine contraction, we have not yet identified its substrates. Identifying substrates for TGs in the uterus and cervix may provide clarity regarding the discrete appearance of TG2 in the uterus. Identifying substrates of the other TG isozymes in the uterus and cervix will also help uncover their roles. The presence and activity of TG4, for example, is of interest because it is known primarily as the prostate TG [7], but was discovered in the rat uterus and cervix in this study.

There is a disparity between the amount of smooth muscle alpha-actin expression in the uterus and cervix [14]. The uterus has significantlymore smooth muscle alpha-actin than the cervix, as visualized qualitatively [14]. The use of contractility experiments answered our question regarding the role of TGs in uterine and cervical contractility, but not the larger question addressing the considerable number of processes TGs could take part of in reproductive tissues.

\section{Limitations}

We recognize several limitations in this study. The activity of TG4, detected in real time RT-PCR and immunohistochemistry, could not be observed in this study because the peptide had not yet been developed at the time of experimentation. In isometric contractility experiments, cystamine does not inhibit specific functions of TG, but the enzyme as a whole [15]. The inhibition of carbamylcholineinduced contraction in the uterus could be caused by inhibition of the GTPase activity, kinase activity, cross- linking ability, or additional post-translational modifications of TG. This study used carbachol as an agonist, and the inhibition of $\mathrm{TG}$ in the uterus should be explored when activated by other agonists. The exact method by which TG contributes to contraction in the uterus is yet unknown.

Finally, the female rats used in this study were not cycled. Signorini et al. have demonstrated increased tissue TG expression during the secretive phase of the menstrual cycle in human endometrium as compared to the proliferative phase, and suggest that expression is selectively increased by exposure to progestagens during the secretive phase [26]. This increased expression did not change the contractility observed in the uteri of our freely cycling rats.

\section{CONCLUSION}

We hypothesized that TGs would be present, active, and functional in the virgin rat uterus and cervix. TG1, TG2, TG3, and TG4 were present in both the uterus and cervix, and TG1 and TG3 were active in the virgin uterus and cervix. The presence of active TG2 in the virgin uterus but not the cervix highlights a discrete difference between these tissues. The TG inhibitor cystamine inhibited carbamylcholine-induced contraction of the virgin uterus but not the cervix, suggesting that TG may play a tissue-specific role in the smooth muscle of the uterus. We provide novel findings of active TG isozymes in both the uterus and cervix. This study further uncovers the differences between the uterus and cervix, and opens new doors to reproductive research.

\section{CONFLICT OF INTEREST}

The authors confirm that this article content has no conflict of interest.

\section{ACKNOWLEDGEMENTS}

Declared none.

\section{REFERENCES}

[1] Lorand L, Graham R. Transglutaminases: crosslinking enzymes with pleiotropic functions. Nat Rev Mol Cell Biol 2003; 4: 140-56.

[2] Facchiano A, Facchiano F. Transglutaminases and their substrates in biology and human disease: 50 years of growing. Amino Acids 2009; 36: 599-614.

[3] Beninati S, Facchiano F, Piacentini M. Transglutaminases: future perspectives. Amino Acids 2012; 44: 1-9.

[4] Ricotta M, Ianuzzi M, Vivo G, Gentile V. Physio-pathological roles of transglutaminase-catalyzed reactions. World J Biol Chem 2010; 1:181-7.

[5] Jeitner T, Pinto J, Krasnikov B, Horswill M, Cooper A. Transglutaminases and neurodegeneration. J. Neurochem 2009; 109: 160-6.

[6] Hitomi K. Transglutaminases in skin epidermis. Eur J Dermatol 2005; 15: 313-9.

[7] Jiang W, Ablin R. Prostate transglutaminase: a unique transglutaminase and its role in prostate cancer. Biomark Med 2011; 5: 285-91

[8] Perez A, Thomas V, Martin G, El Alaoui S. Identification of human salivary transglutaminases. Amino Acids 2011; 44: 245-50.

[9] Johnson K, Peterson-Jones H, Thompson J et al. Vena cava and aortic smooth muscle cells express transglutaminase 4 in addition to transglutaminase 2. Am J Physiol Heart Circ Physiol 2012; 302: $1355-66$ 
[10] Aguilar H, Mitchell B. Physiological pathways and molecular mechanisms regulating uterine contractility. Hum Reprod Update 2010; 16: 725-44.

[11] Sugimura $\mathrm{Y}$, Hosono $\mathrm{M}$, Kitamura $\mathrm{M}$ et al. Identification of preferred substrate sequences for transglutaminase 1 - development of a novel peptide that can efficiently detect cross-linking enzyme activity in the skin. FEBS J 2008; 275: 5667-77.

[12] Sugimura Y, Ueda H, Maki M, Hitomi K. Novel site-specific immobilization of a functional protein using a preferred substrate sequence for transglutaminase 2. J Biotechnol 2007; 131: 121-7.

[13] Yamane A, Fukui M, Sugimura $Y$ et al. Identification of a preferred substrate peptide for transglutaminase 3 and detection of in situ activity in skin and hair follicles. Eur J Biochem 2012; 277: 3564-74.

[14] Darios E, Seitz B, Watts SW. Smooth muscle pharmacology in the isolated virgin and pregnant rat uterus and cervix. J Pharmacol Exp Ther 2012; 341: 587-96.

[15] Jeitner T, Delikatny J, Ahlqvist J, Capper H, Cooper A. Mechanism for the inhibition of transglutaminase 2 by cystamine. Biochem Pharmacol 2005; 69: 961-70.

[16] Siegel M, Khosla C. Transglutaminase 2 inhibitors and their therapeutic role in disease states. Pharmacol Ther 2007; 115: 23245.

[17] Jarvinen A, Paakkari I. Carbachol differs from classical muscarinic agonists in producing long-term desensitization in smooth muscle. Arch Int Pharmacodyn Ther 1991; 310:66-75.
[18] Sato Y, Hotta H, Nakayama H, Suzuki H. Sympathetic and parasympathetic regulation of the uterine blood flow and contraction in the rat. J Auton Nerv Syst 1996; 59: 151-8.

[19] Ward R. Foundations for Osteopathic Medicine. New York: Lippincott Williams and Wilkins 2003.

[20] Iismaa S, Mearns B, Lorand L, Graham R. Transglutaminases and disease: lessons from genetically engineered mouse models and inherited disorders. Physiol Rev 2009; 89: 991-1023.

[21] Sizemore N, Kasturi L, Gorodeski G, Eckert R, Jetten A, Rorke E Retinoid regulation of human ectocervical epithelial cell transglutaminase activity and keratin gene expression. Differentiation 1993; 54: 219-25.

[22] Friedrich M, Villena-Heinsen $\mathrm{C}, \mathrm{He} \mathrm{J}$ et al. Expression of transglutaminase $\mathrm{K}$ in normal cervix tissue and cervix carcinomas. Histochem J 1999; 31: 13-8.

[23] Del Nonno F, Pisani G, Visca P et al. Role and predictive strength of transglutaminase type 2 expression in premalignant lesions of the cervix. Mod Pathol 2011; 24: 855-65.

[24] Alcock J, Warren A, Goodson Y, Hill S, Khan R, Lymn J. Inhibition of tissue transglutaminase 2 attentuates contractility of pregnant human myometrium. Biol Reprod 2011; 84: 646-53.

[25] Del Campo L, Tuna B, Ferrer M, Bavel E, Bakker E. Testerone and $\beta$-oestradiol prevent inward remodeling of rat small mesenteric arteries: role of NO and transglutaminase. Clinical Science 2013; 124: 719-28.

[26] Signorini M, Pasini F, Bonaccorsi G, Mollica G, Ferrari C, Bergamini C. Regulation of endometrial transglutaminase activity during the menstrual cycle. Biochem Int 1988; 16: 77-82.

(C) Young et al.; Licensee Bentham Open.

This is an open access article licensed under the terms of the Creative Commons Attribution Non-Commercial License (http://creativecommons.org/licenses/by-nc/3.0/) which permits unrestricted, non-commercial use, distribution and reproduction in any medium, provided the work is properly cited. 